\title{
OPEN The influences of low protein diet on the intestinal microbiota of mice
}

\author{
Hiroaki Masuoka ${ }^{1 凶}$, Wataru Suda ${ }^{2}$, Eriko Tomitsuka ${ }^{3}$, Chie Shindo ${ }^{2}$, Lena Takayasu ${ }^{2,4}$, \\ Paul Horwood ${ }^{5,6}$, Andrew R. Greenhill ${ }^{6,7}$, Masahira Hattori ${ }^{2,8}$, Masahiro Umezaki ${ }^{4}$ \& \\ Kazuhiro Hirayama ${ }^{1}$
}

Recent research suggests that protein deficiency symptoms are influenced by the intestinal microbiota. We investigated the influence of low protein diet on composition of the intestinal microbiota through animal experiments. Specific pathogen-free (SPF) mice were fed one of four diets $(3,6,9$, or $12 \%$ protein) for 4 weeks ( $n=5$ per diet). Mice fed the $3 \%$ protein diet showed protein deficiency symptoms such as weight loss and low level of blood urea nitrogen concentration in their serum. The intestinal microbiota of mice in the $3 \%$ and $12 \%$ protein diet groups at day 0,7 , 14,21 and 28 were investigated by $16 \mathrm{~S}$ rRNA gene sequencing, which revealed differences in the microbiota. In the $3 \%$ protein diet group, a greater abundance of urease producing bacterial species was detected across the duration of the study. In the $12 \%$ diet protein group, increases of abundance of Streptococcaceae and Clostridiales families was detected. These results suggest that protein deficiency may be associated with shifts in intestinal microbiota.

Protein is an indispensable structural and functional cellular component and is essential for survival in all organisms ${ }^{1}$. Even when energy intake is sufficient, protein deficiency causes growth retardation and loss of muscle mass. Moreover, protein deficiency increases susceptibility to infection and induces fatty liver ${ }^{2-4}$, and can lead to fatigue, diarrhea, and edema ${ }^{3,5}$. In short, consuming a protein-deficient diet is detrimental to health and can be life-threatening ${ }^{6,7}$. Low protein levels can be detected by low serum albumin (Alb) and total protein (TP) concentrations, and a decrease in serum albumin-globulin $(\mathrm{A} / \mathrm{G}) \mathrm{ratio}^{6,8}$ in humans.

Recent research suggests that the onset of kwashiorkor, a disorder believed to be prevalent among children who consume a low protein diet, is related to the composition and function of the intestinal microbiota. Smith et al. argued that kwashiorkor is caused by a combination of "pathogenic" bacteria and a nutritionally inadequate diet in Malawi ${ }^{9}$. This suggests that children with "favorable" intestinal microbiota may not develop kwashiorkor even when they consume a nutritionally deficient diet. Following the same cohorts, Blanton et al. showed that Ruminococcus gnavus and Clostridium symbiosum ameliorated the impaired growth phenotype of children in Malawi ${ }^{10}$. These studies suggest that the intestinal microbiota transition during protein deficiency is associated with host homeostasis. However, the relationship between microbiota and host nitrogen balance is not fully understood. Therefore, we investigated the effects of protein deficiency on the intestinal microbiota to elucidate the potential roles of intestinal bacteria on the host nutrition.

A previous report described changes in bacterial species abundance in gnotobiotic mice inoculated with 10 gut bacterial species due to composition of diet ${ }^{11}$. However, few studies have investigated the response of the intestinal microbiota to a low protein diet. In this study, we investigated the influence of a low protein diet on nutritional status and intestinal microbiota composition. We fed specific pathogen-free (SPF) mice diets with different protein concentrations to investigate changes in nutritional status (i.e., food and water consumption and body weight gain), serum biomarkers (i.e., Alb, TP, A/G ratio, and blood urea nitrogen (BUN)) and the composition of intestinal microbiota between 3 and 12\% protein diet groups at days 0, 7, 14, 21 and 28 .

${ }^{1}$ Department of Veterinary Medical Science, Graduate School of Agricultural and Life Sciences, The University of Tokyo, Tokyo, Japan. ${ }^{2}$ RIKEN Center for Integrative Medical Sciences, Yokohama, Japan. ${ }^{3}$ Department of Health Chemistry, Faculty of Pharmaceutical Sciences, Niigata University of Pharmacy and Applied Sciences, Niigata, Japan. 'Department of Human Ecology, Graduate School of Medicine, The University of Tokyo, Tokyo, Japan. ${ }^{5}$ College of Public Health, Medical and Veterinary Sciences, James Cook University, Townsville, Australia. ${ }^{6}$ Papua New Guinea Institute of Medical Research, Goroka, Papua New Guinea. ${ }^{7}$ School of Science, Psychology and Sport, Federation University Australia, Churchill, Australia. ${ }^{8}$ Graduate School of Advanced Science and Engineering, Waseda University, Tokyo, Japan. ${ }^{\circledR e m a i l: ~ h i r o a k i-m a s u o k a @ g . e c c . u-t o k y o . a c . j p ; ~ w a t a r u . s u d a @ ~}$ riken.jp 


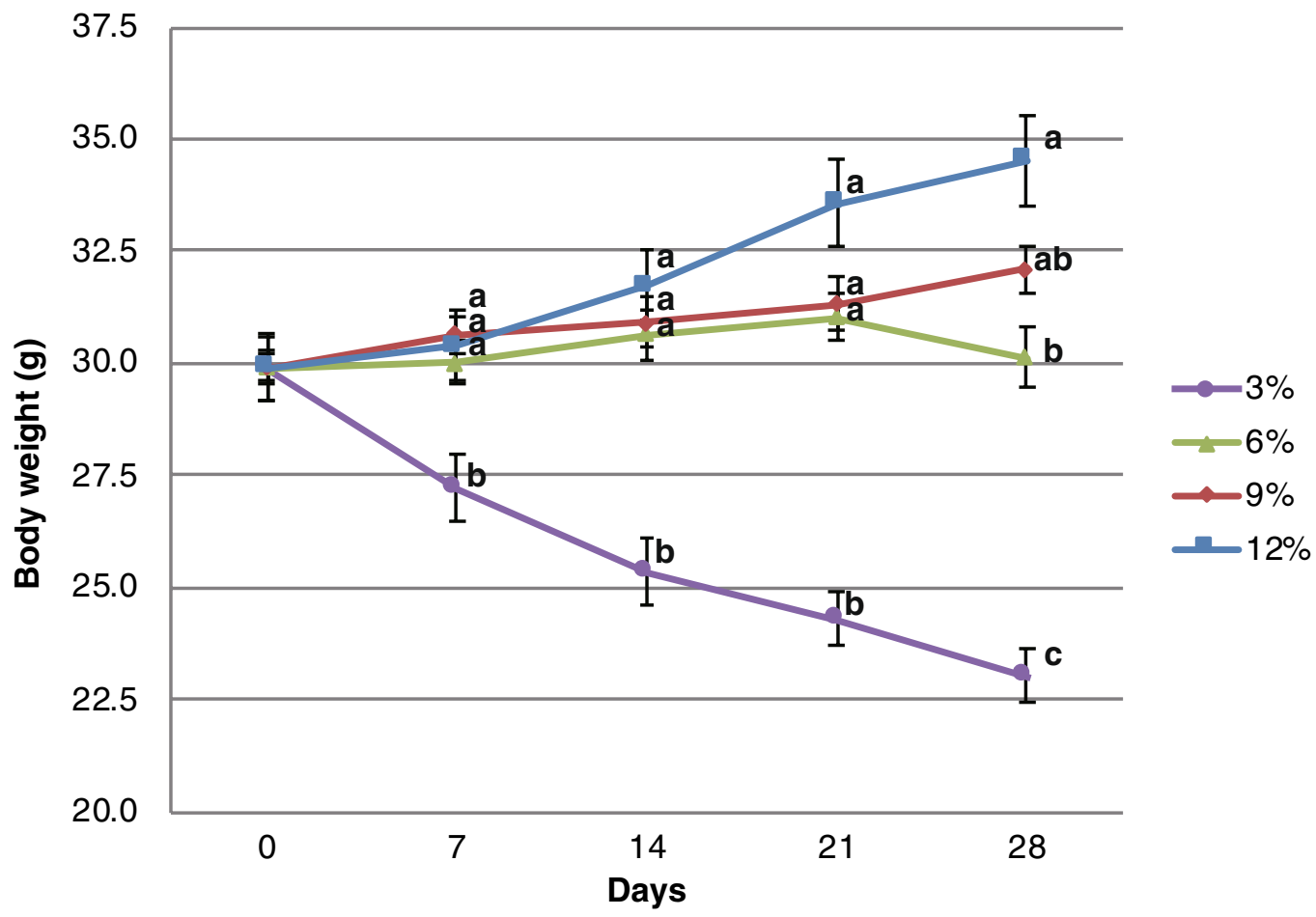

Figure 1. Line graph of changes in raw body weight over 4 weeks of the second experiment. Each group of mice $(n=5)$ was fed a diet with different protein concentration $(3,6,9$, and $12 \%)$. The mean and standard error of the mean (SEM) are shown. Plots indicated with the same letters $(\mathbf{a}, \mathbf{b}$ and $\mathbf{c})$ on the same days were not significantly different at $P<0.05$ by Tukey-Kramer test.

\section{Results}

Body weight, diet and water consumption. Body weight and food and water consumption were recorded in two independent experiments: one set up to investigate nutritional impact of protein in diet (first experiment) and the other focusing on correlations between protein content in diet and the intestinal microbiota of mice (second experiment).

A line graph of the raw body weight of each group in the first experiment can be found as Supplementary Figure S1. In the first experiment, the body weight of the 3\% protein diet group was significantly lower than those of the $6 \%$ group on day $28(P=0.048$ by Tukey-Kramer test $)$, those of the $9 \%$ group from day $14(P=0.028,0.038$ and 0.013 on day 14,21 and 28, respectively, by Tukey-Kramer test) and $12 \%$ protein diet groups from day 14 $(P=0.0034,0.0026$ and 0.0002 on day 14,21 and 28 , respectively, by Tukey-Kramer test). There were no significant differences between the body weights of the $6 \%, 9 \%$ and $12 \%$ groups throughout the experimental period. The relative body weight of the $3 \%$ protein diet group decreased to $96.4 \pm 2.5 \%$ on day 28 , whereas the relative body weight of the $12 \%$ protein diet group increased to $120.6 \pm 2.5 \%$. A line graph of the raw body weight of each group in the second experiment can be found in Fig. 1. In the second experiment, the body weight data have similar tendency to the data of the first experiment. The body weight of the $3 \%$ protein diet group in the second experiment was significantly lower than those of the $6 \%$ group $\left(P=0.0304,0.0002,2.67 \times 10^{-5}\right.$ and $2.04 \times 10^{-5}$ on day 7, 14, 21 and 28, respectively, by Tukey-Kramer test), $9 \%$ group $\left(P=0.0073,0.0001,1.52 \times 10^{-5}\right.$ and $9.14 \times 10^{-7}$ on day $7,14,21$ and 28 , respectively, by Tukey-Kramer test $)$ and $12 \%$ protein diet groups $\left(P=0.0131,2.30 \times 10^{-5}\right.$, $4.26 \times 10^{-7}$ and $3.48 \times 10^{-8}$ on day 7,14, 21 and 28 , respectively, by Tukey-Kramer test). There were no significant differences between the body weights of the $6 \%, 9 \%$ and $12 \%$ protein diet groups throughout the experimental period. The relative body weight of the $3 \%$ protein diet group decreased to $77.1 \pm 2.1 \%$ on day 28 , whereas the relative body weight of the $12 \%$ protein diet group increased to $115.4 \pm 2.0 \%$.

The amount of water and food consumed by each diet group was recorded for both the nutrition and the microbiota study (Supplementary Figure S2). In the first experiment, no difference in food consumption was observed among the four groups (Figure S2a); however, water consumption was higher in the 3\% protein diet group than in other groups (Figure S2b). A line graph of the amount of water and food consumption of each group in the second experiment can be found as Fig. 2. In the second experiment, food consumption was again similar across all diet groups (Fig. 2a), and water consumption was again higher in the $3 \%$ protein group (Fig. 2b). The amount of water consumed by the $3 \%$ protein diet group was more than twice that of the $12 \%$ protein diet group at day 28 .

Serum biomarkers. The bar graphs of serum concentrations can be found as Supplementary Figure S3. At the end of the experiment (day 28) the 3\%,6\%, and $9 \%$ protein diet groups had significantly lower serum BUN 
a

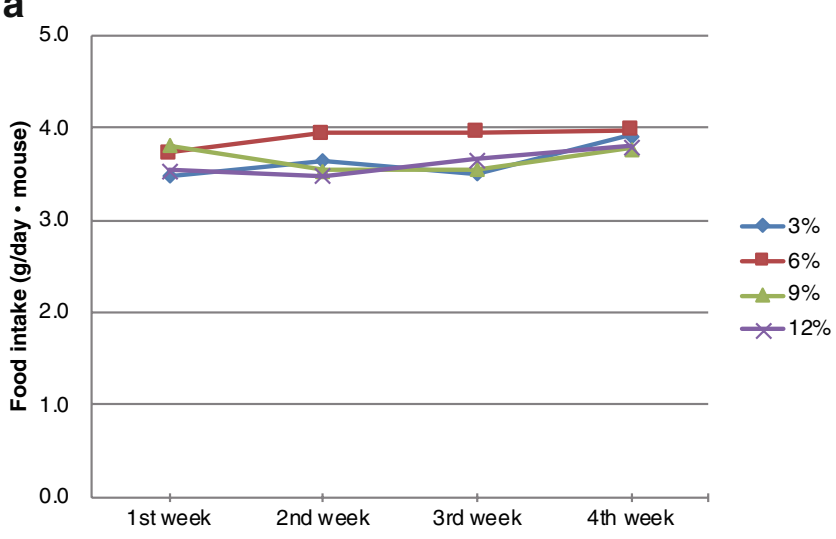

b

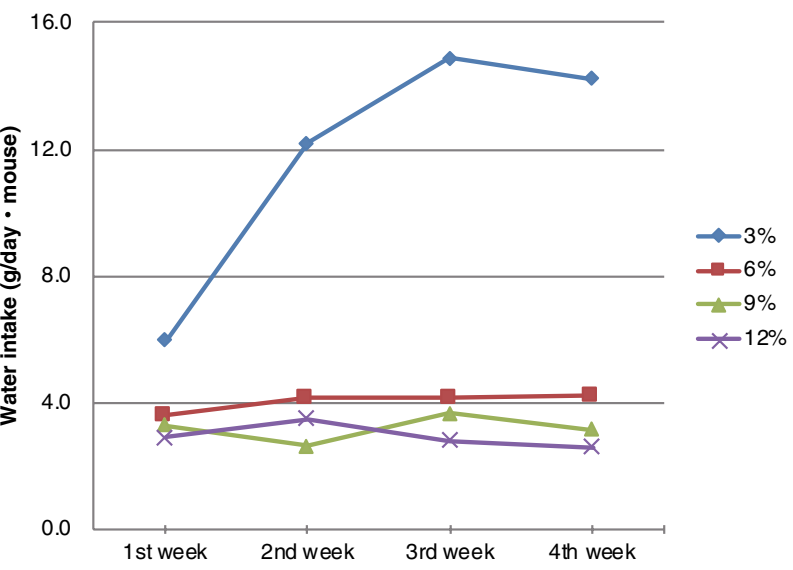

Figure 2. Food and water intakes of mice fed different protein diets in the second experiment. Mean values for each week are shown. "1st week" means the period from day 1 to day 7, "2nd week" means the period from day 8 to day 14, "3rd week" means the period from day 15 to day 21 and "4th week" means the period from day 22 to day 28. (a) Consumption of food per day (average) in mice according to protein diet. (b) Consumption of water per day (average) in mice according to protein diet. Differences in water and food consumption between mice fed different diets could not be investigated with statistical tests because they were evaluated by cage, thus individual variation among mice could not be evaluated.

than those in the $12 \%$ protein diet group $\left(P=1.77 \times 10^{-5}, 0.006\right.$ and 0.002 by Tukey-Kramer test, respectively). The serum BUN concentrations at day 28 in the $3 \%$ protein diet group were also significantly lower than that in the $6 \%$ protein diet groups ( $P=0.034$ by Tukey-Kramer test) (Figure S3a). There were no significant differences in serum concentrations of albumin, total protein and A/G at day 28 among any of the groups (Figure S3b, S3c and S3d).

Diversity of the intestinal microbiota. Figure 3 shows box plots of four a diversity indices of the intestinal microbiota in the $3 \%$ and $12 \%$ protein diets on day $0,7,14,21$ and 28 . The OTU number, Chaol, abundancebased coverage estimator (ACE) and Shannon's index did not differ throughout the experiment in the 3\% protein diet group, whereas Chao 1 and ACE were significantly lower on day 28 than on day 0 in the $12 \%$ protein diet group ( $P=0.032$ by Wilcoxon rank sum test and Benjamini-Hochberg $P$ value correction). Shannon's index in the $12 \%$ protein diet group on day $7,14,21$ and 28 were significantly lower than that on day 0 . Principal coordinates analysis (PCoA) was performed on both weighted and unweighted UniFrac distances of intestinal microbiota composition of the mice in the $3 \%$ and $12 \%$ diet groups throughout the experiment (Fig. 4). Permutational multivariate analysis of variance (PERMANOVA) in the $3 \%$ protein diet group based on weighted UniFrac distance revealed that there were significant dissimilarities between day 0 and the other days $\left(\mathrm{R}^{2}=0.840\right.$, $P=0.011$ between day 0 and day $7 ; \mathrm{R}^{2}=0.916, P=0.011$ between day 0 and day $14 ; \mathrm{R}^{2}=0.921, P=0.011$ between day 0 and day $21 ; \mathrm{R}^{2}=0.807, P=0.011$ between day 0 and day 28). Furthermore, PERMANOVA in the $12 \%$ diet group based on weighted UniFrac distance revealed that there were also significant dissimilarities between day 0 and the other days $\left(\mathrm{R}^{2}=0.795, P=0.021\right.$ between day 0 and day $7 ; \mathrm{R}^{2}=0.861, P=0.021$ between day 0 and day 14; $\mathrm{R}^{2}=0.769, P=0.021$ between day 0 and day $21 ; \mathrm{R}^{2}=0.861, P=0.021$ between day 0 and day 28 ). The dissimilarities on weighted UniFrac distances between the $3 \%$ and $12 \%$ protein diet groups at each day are shown in Supplementary Figure S4, while Supplementary Figure S5 shows a PCoA plot based on unweighted UniFrac distance.

Compositions of the intestinal microbiota. Microbial composition analyses were conducted at phylum level for phyla present at an abundance $>0.1 \%$ in at least one diet group and compared for different time points (Fig. 5a). Outcomes of statistical analyses, using Wilcoxon rank sum test with Benjamini-Hochberg $P$ value procedure, are provided in Supplementary Table S1a and S1b, with a summary of key findings provided below. In the $3 \%$ protein diet group, the proportions of Actinobacteria were significantly higher on day 14, 21 and 28 than on day $0(P=0.032,0.032$ and 0.032 , respectively). The proportions of Bacteroidetes were significantly lower on day $7,14,21$ and 28 than on day $0(P=0.032,0.032,0.032$ and 0.032 , respectively). The proportions of Firmicutes were significantly higher on day 7 than on day $0(P=0.032)$, whereas those on day 14,21 and 28 were significantly lower than on day $0(P=0.032,0.032$ and 0.032 , respectively). The proportions of Proteobacteria were significantly higher on day 14,21 and 28 than on day $0(P=0.048,0.032$ and 0.048 , respectively). The proportions of Tenericutes were significantly lower on day 28 than on day $0(P=0.032)$. On the other hand, in the $12 \%$ protein diet group, the proportions of Bacteroidetes were significantly lower on day 14 and 21 than on day $0(P=0.032$ and 0.032 , respectively). The proportions of Firmicutes were significantly higher on day 14 and 21 than on day $0(P=0.032$ and 0.032 , respectively). The proportions of Proteobacteria were significantly higher on day $7,14,21$ and 28 than on day $0(P=0.048,0.032,0.032$ and 0.032 , respectively). The proportions of Actinobacteria were not significantly changed throughout the experiment. In addition to $P$ values, Tables S1a and $1 \mathrm{~b}$ provide average read numbers in each group throughout the experiment for these phyla. 

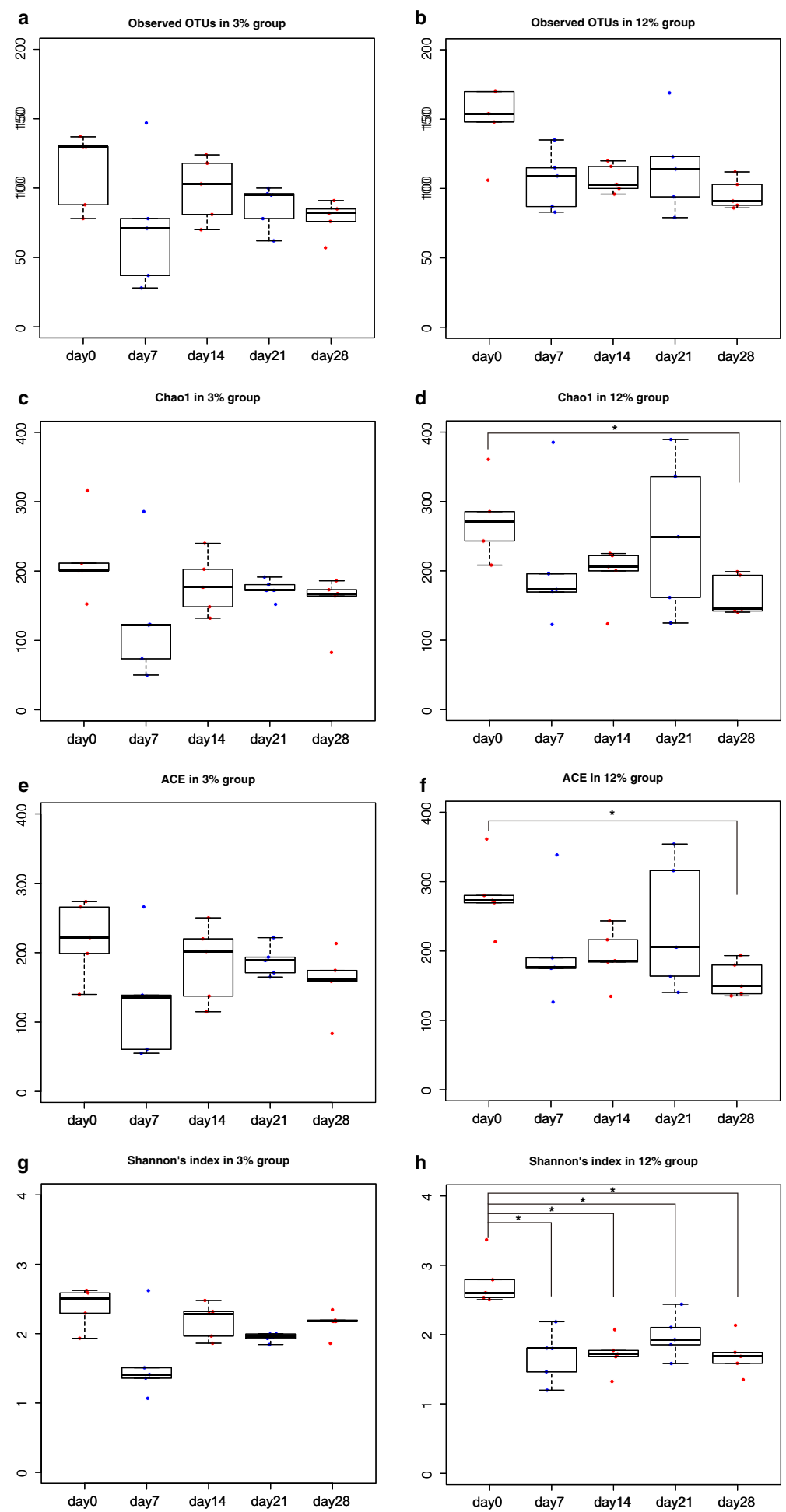

Figure 3. Measures of a-diversity of intestinal microbiota of mice fed $3 \%$ and $12 \%$ protein diet. The label of data on day $0,7,14,21$ and 28 , were shown as day 0 , day7, day14, day21 and day28 respectively. The symbols * indicates $P<0.05$. (a) The box plots of observed Operational taxonomic unit (OTU) number of the intestinal microbiota of mice in the $3 \%$ protein group. (b) The box plots of observed OTU number of the intestinal microbiota of mice in $12 \%$ protein group. (c) The box plots of Chao 1 in the $3 \%$ protein group. (d) The box plots of Chao1 in the $12 \%$ protein group. (e) The box plots of ACE in the 3\% protein group. (f) The box plots of ACE in the $12 \%$ protein group. (g) The box plots of Shannon's index in the 3\% protein group. (h) The box plots of Shannon's index in the $12 \%$ protein group. 
a

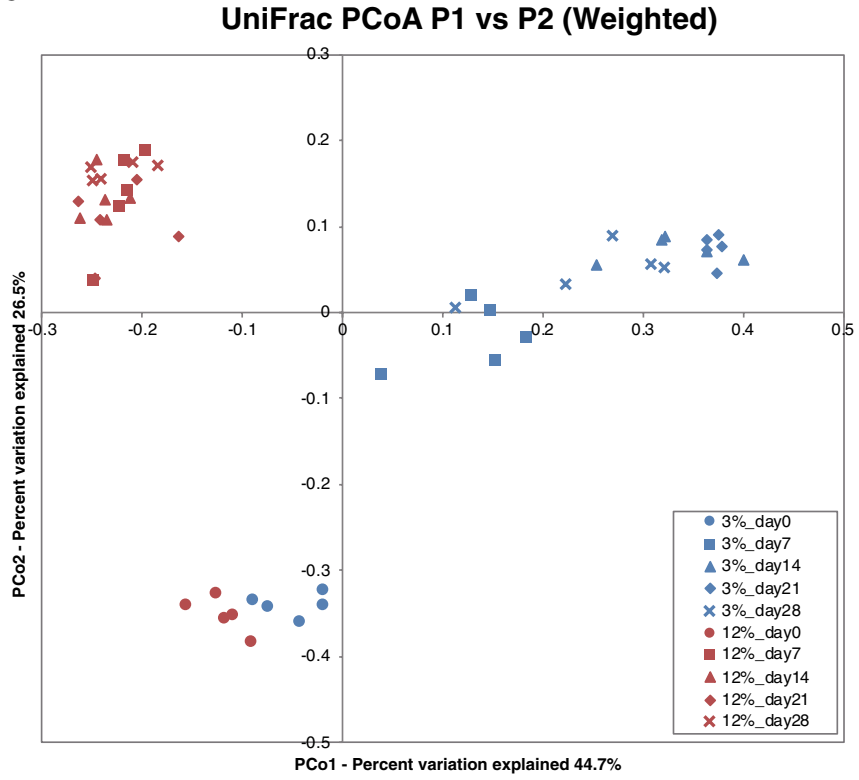

b

\begin{tabular}{|c|c|c|c|c|}
\hline \multicolumn{2}{|c|}{ Category } & \multirow{2}{*}{ No. subject } & \multirow{2}{*}{$\frac{\mathbf{R}^{\mathbf{2}}}{0.840}$} & \multirow{2}{*}{$\frac{\text { adjusted } \mathrm{p} \text {-value }}{0.011}$} \\
\hline \multirow{4}{*}{$3 \%$ day 0} & vs $3 \% \_$day 7 & & & \\
\hline & vs $3 \%$ _day 14 & \multirow{9}{*}{5 in each group } & 0.916 & 0.011 \\
\hline & vs $3 \% \_$day 21 & & 0.921 & 0.011 \\
\hline & vs 3\%_day 28 & & 0.807 & 0.011 \\
\hline \multirow{3}{*}{$3 \%$ day 7} & vs $3 \%$ _day 14 & & 0.770 & 0.011 \\
\hline & vs $3 \%$ _day 21 & & 0.841 & 0.011 \\
\hline & vs $3 \%$ _day 28 & & 0.768 & 0.011 \\
\hline \multirow{2}{*}{ 3\%_day 14} & vs $3 \%$ day 21 & & 0.436 & 0.015 \\
\hline & vs 3\%_day 28 & & 0.519 & 0.011 \\
\hline 3\%_day 21 & vs $3 \%$ _day 28 & & 0.440 & 0.011 \\
\hline \multicolumn{2}{|c|}{ Category } & No. subject & $\mathbf{R}^{2}$ & adjusted p-value \\
\hline \multirow{4}{*}{$12 \%$ _day 0} & vs $12 \%$ day 7 & \multirow{10}{*}{5 in each group } & 0.795 & 0.021 \\
\hline & vs $12 \%$ _day 14 & & 0.861 & 0.021 \\
\hline & vs $12 \%$ day 21 & & 0.769 & 0.021 \\
\hline & vs $12 \%$ day 28 & & 0.861 & 0.021 \\
\hline \multirow{3}{*}{$12 \%$ _day 7} & vs $12 \%$ day 14 & & 0.036 & 0.907 \\
\hline & vs $12 \%$ day 21 & & 0.098 & 0.629 \\
\hline & vs $12 \%$ day 28 & & 0.063 & 0.760 \\
\hline \multirow{2}{*}{$12 \%$ day 14} & vs $12 \%$ day 21 & & 0.141 & 0.371 \\
\hline & vs $12 \%$ day 28 & & 0.063 & 0.371 \\
\hline $12 \%$ day 21 & vs $12 \%$ day 28 & & 0.133 & 0.371 \\
\hline
\end{tabular}

Figure 4. Measures of $\beta$-diversity of intestinal microbiota of mice fed $3 \%$ and $12 \%$ protein diet. (a) Principal coordinates analysis (PCoA) plot based on the weighted UniFrac distances for intestinal microbiota composition in mice in each group in the second experiment. Plots of the $3 \%$ protein group were shown in blue and those of the $12 \%$ protein group were shown in red. (b) Evaluation of dissimilarity of the $3 \%$ protein group by PERMANOVA in the analysis of weighted UniFrac distance. $\mathrm{R}^{2}$ indicates coefficient of determination. Significant adjusted $P$ values are in bold. (c) Evaluation of dissimilarity of the $12 \%$ protein group by PERMANOVA in the analysis of weighted UniFrac distance. $\mathrm{R}^{2}$ indicates coefficient of determination. Significant adjusted $P$ values are in bold.

a

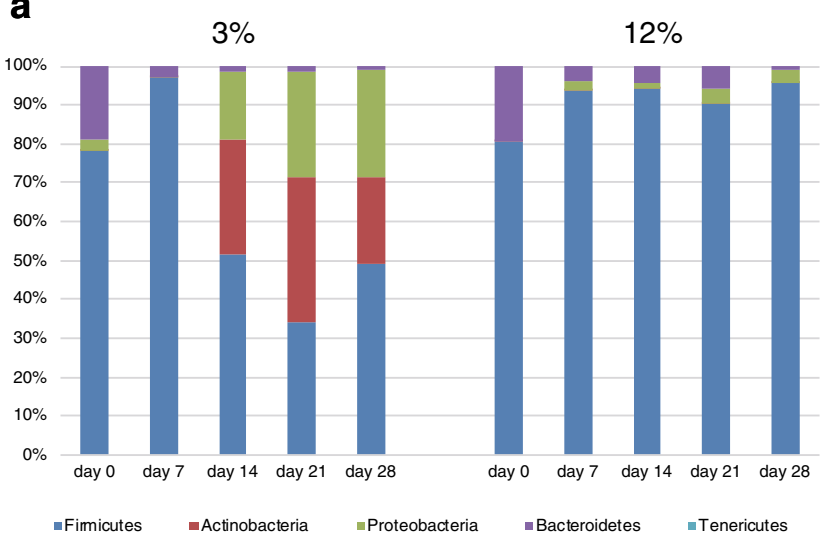

b

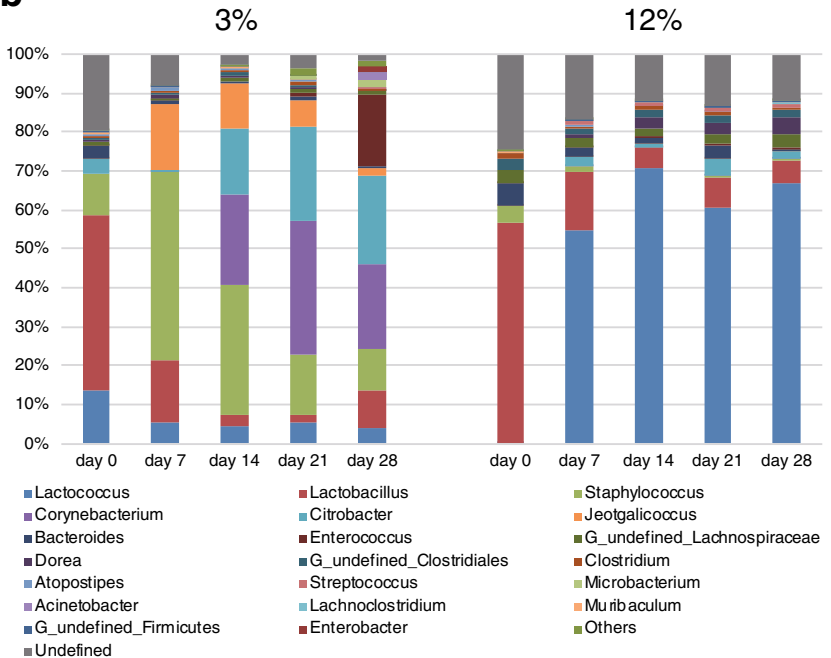

Figure 5. Predominant taxa by relative abundance detected in mice faeces from mice fed $3 \%$ protein diet and $12 \%$ protein diet. (a) Relative abundance of composition of intestinal microbiota at the phylum level across 4 weeks. (b) Relative abundance of composition of intestinal microbiota at the genus level across 4 weeks.

Analysis was also conducted at genus level for genera with $>0.1 \%$ abundance in at least one diet group (Fig. $5 \mathrm{~b}$ ). In the $3 \%$ protein diet group, there were bacteria whose relative abundance increased or decreased throughout the experiment. Outcomes of statistical analyses, using Wilcoxon rank sum test with Benjamini-Hochberg $P$ value procedure are provided in Supplementary Table S1c, and summarized below. The proportions of Acinetobacter, Enterobacter and Enterococcus were significantly higher on day 28 than those on day $0(P=0.032$ for all three genera). The proportions of Microbacterium were significantly higher on day 21 and 28 than that on day $0(P=0.032$ and 0.032$)$. The proportions of Corynebacterium were significantly higher on day 14,21 and 28 than that on day $0(P=0.032,0.032$ and 0.032$)$. The proportions of Jeotgalicoccus were significantly 


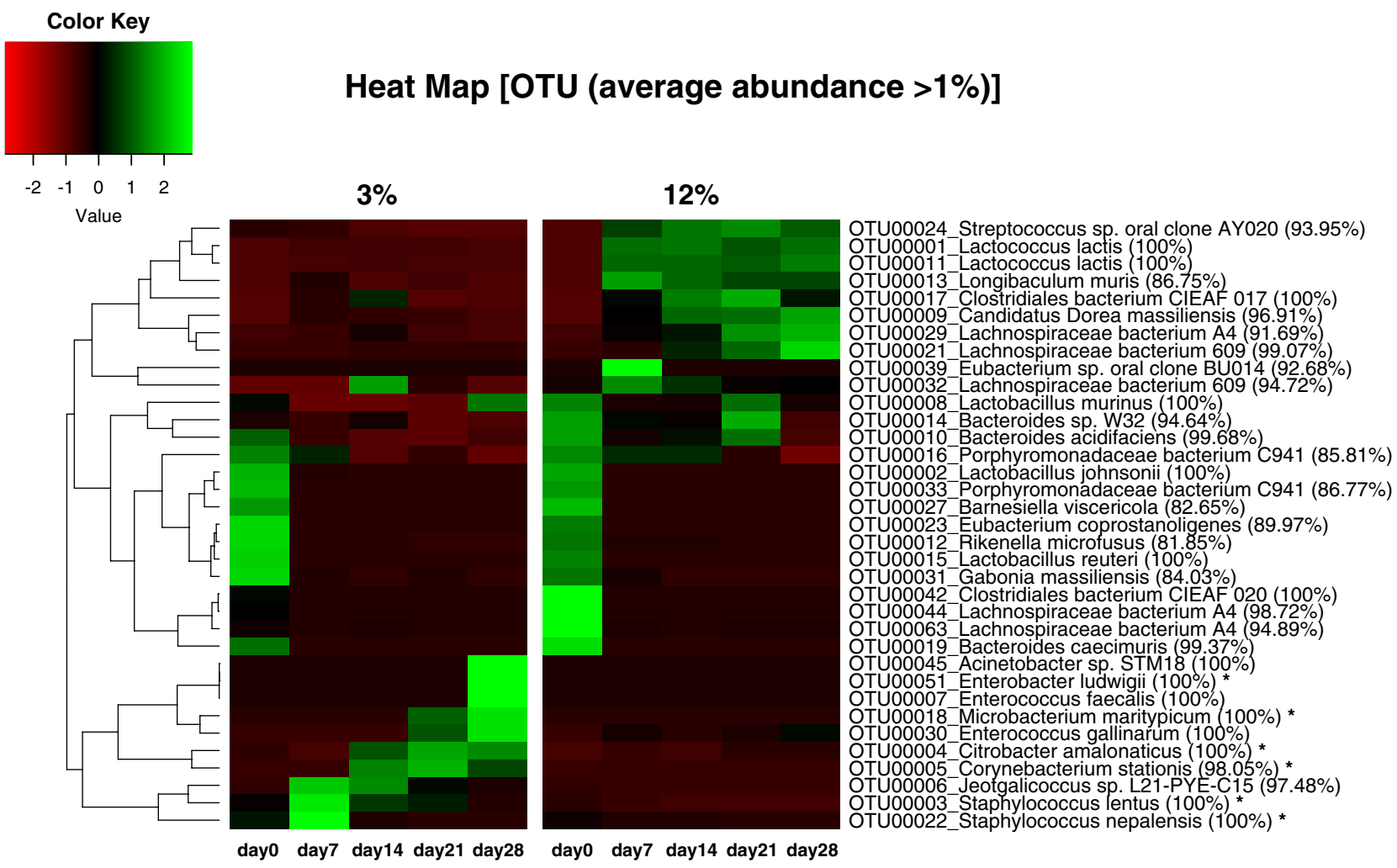

Figure 6. Heatmap of the phylotypes assigned to species-level taxa for OTUs with more than $1 \%$ abundance in at least 1 group. The color in the heatmap reflects the z-score; green shows that the ratio of OTUs are high in each group, and red shows that that are low in each group. The symbols * indicates OTUs assigned to ureasepositive bacteria.

higher on days 7, 14, 21 and 28 than those on day $0(P=0.032,0.032,0.032$ and 0.032$)$. The proportions of Staphylococcus were significantly higher on day 7 than that on day $0(P=0.032)$. The proportions of Citrobacter were significantly higher on day 14 and 21 than that on day $0(P=0.048$ and 0.032 , respectively). The proportions of Lactobacillus, Lactococcus and Muribaculum were significantly higher on day 0 than those on days 7, 14, 21 and $28(P=0.032,0.032,0.032$ and 0.032 for all three genera $)$ and the proportions of Bacteroides also changed across the four sample days $(P=0.048,0.048,0.032$ and 0.048 , respectively). The proportions of Dorea were significantly higher on day 0 than those on day 14,21 and $28(P=0.048,0.048$ and 0.032 , respectively). In the $12 \%$ protein diet group, there were also bacteria whose relative abundance increased or decreased throughout the experiment. The proportions of Dorea, Lactococcus and Streptococcus were significantly lower on day 0 than on days $7,14,21$ and $28(P=0.032,0.032,0.032$ and 0.032 for all four genera). The proportions of Citrobacter $(P=0.048$, $0.032,0.032$ and 0.032 , respectively) and Enterococcus $(P=0.048,0.048,0.048$ and 0.032 , respectively) changed in the same way over days 7, 14, 21 and 28. The proportions of Lactobacillus, Muribaculum and Staphylococcus were significantly higher on day 0 than on days $7,14,21$ and $28(P=0.032,0.032,0.032$ and 0.032 for all three genera). The proportions of Atopostipes were significantly higher on day 0 than that on day 21 and $28(P=0.048$ and 0.032 , respectively). The proportions of Bacteroides were significantly higher on day 0 than that on day 28 $(P=0.032)$. Average read numbers in each group and $P$ values between day 0 and the other each day of these genera can be found in Supplementary Table S1c and $1 \mathrm{~d}$.

Figure 6 provides a heatmap which represents abundance of the selected OTUs with more than $1 \%$ abundance in at least one diet group average from day 0 to day 28 in either groups. The read numbers of OTUs shown in Fig. 6 and their $P$ values between day 0 and the other each day in each group can be found as Supplementary Table S2. The total average abundance of these selected OTUs accounted for $90.4 \pm 1.10 \%$.

\section{Discussion}

In the present study, we investigated the effects of low protein diet on the composition of intestinal microbiota and nutritional status in male SPF mice. We used only male mice to remove sex-related effects thus ensuring the analysis was focused on the intestinal microbiota change related to differences in nutrition. Mice fed a $3 \%$ protein diet showed signs of protein deficiency, such as growth retardation and low serum BUN. The consistent food intake throughout the experiment suggests that weight loss observed in the $3 \%$ protein group was not caused by a decrease in the amount of food consumed, as the amount of food consumed did not differ amongst the four dietary groups. Interestingly, the amount of water consumption by the $3 \%$ protein group was considerably higher than that consumed by the other groups. Other researchers have investigated food intake in low-protein diets, but they provided inconsistent results. Our results on food consumption corresponded with the findings of 
Larson et al. ${ }^{12}$, but are contrary to the other research in which an increase in food consumption was reported ${ }^{13,14}$. Inconsistent results across numerous studies are difficult to fully explain, but may reflect differences in age, sex and breed. In the current study, we demonstrated that mice fed a low protein intake consumed more water. To our knowledge, this is the first report of protein intake correlating with water intake.

Previous reports indicated that the plasma albumin level of rats tended to decrease at some time points when they were fed low protein $\operatorname{diet}^{15,16}$. On the other hand, Wada et al. indicated that there was no difference in plasma albumin between control groups and low protein groups after 4 weeks ${ }^{16}$. Hubert et al. also revealed that there was almost no difference in plasma albumin between ad libitum group and diet-restricted group after 4 weeks ${ }^{17}$. In agreement with these previous studies, we also showed that plasma albumin concentration among mice fed a low protein diet did not decrease over the 4 weeks of our study.

In the present study, there were differences in gut microbial composition between the $3 \%$ and $12 \%$ groups at day 0 , yet the dissimilarity on UniFrac distances between the $3 \%$ and $12 \%$ protein diet groups at day 0 were significantly lower than those of the other time points (Supplementary Figure S4). Thus, it is regarded that the variations detected in this study were mostly affected by dietary changes, though the difference at day 0 might be related to cage-specific effects. These microbiota shifts accompanied by dietary changes potentially include not only differences in nutrition but also host systemic changes such as weight loss. In reaction to the $3 \%$ protein diet, the proportions of 10 predominant OTUs assigned to Staphylococcus lentus, Citrobacter amalonaticus, Enterococcus faecalis, Jeotgalicoccus sp., Corynebacterium stationis, Staphylococcus nepalensis, Enterobacter ludwigii, Acinetobacter sp., Enterococcus gallinarum and Microbacterium maritypicum increased. Among these OTUs, 6 OTUs including E. ludwigii, M. maritypicum, C. amalonaticus, C. stationis, S. lentus and S. nepalensis have been previously reported to have ureases, the enzymes catalyzing the hydrolysis of urea into carbon dioxide and ammonia ${ }^{18-23}$. It was reported that urea in the gastrointestinal tract excreted from epithelial cells is cleaved into ammonia and carbon dioxide when sufficient urease is produced by intestinal bacteria ${ }^{24}$, and some bacteria utilize ammonia to produce amino acids and peptides ${ }^{25}$. The host can absorb either the amino acids or the peptides synthesized by the bacteria. This process is called "urea nitrogen salvage" caused by urease-producing and ammonia-utilizing bacteria. It is also known that the absorption of ammonia produced from urea and its recycling into amino acids occur at the host's systemic levels ${ }^{26}$. Further studies would be necessary to clarify the contribution of microbiota to host's nitrogen balances.

In reaction to the $12 \%$ protein diet, the proportion of 8 predominant OTUs assigned to Streptococcus sp., Lactococcus lactis (2 OTUs), Longibaculum muris, Clostridiales bacterium, Candidatus Dorea massiliensis and Lachnospiraceae bacterium (2 OTUs) increased. Among these 8 OTUs, 3 OTUs assigned to L. lactis and Streptococcus sp. belong to the Streptococcaceae family, and the other 5 out of 8 OTUs assigned to the Clostridiales bacterium, Candidatus Dorea massiliensis and Lachnospiraceae bacterium belong to the Lachnospiraceae family. OTUs assigned to Streptococcaceae family increased even in the $3 \%$ protein group, but showed significant increase in the $12 \%$ protein group. The high proportion of L. lactis in the $12 \%$ protein diet group may have been due to composition of the diet. The protein sources of the NMF diet used for acclimatization are plant and animal based, i.e., soybeans and fish powders; whereas those of the $3 \%$ and $12 \%$ protein diets are caseins (from mammalian milk). A previous report by Bisanz et al. ${ }^{27}$ suggested that Lactococcus species might be favored by casein in experimental diets.

Several limitations should be discussed. First, we did not set a control group by feeding commercial solid diet (containing $27.5 \%$ of protein), although that was used for acclimatization. Since our purpose was to compare the impact of "a diet containing a sufficient amount of protein" and "protein deficient diets", we judged that a commercial diet contains an excess amount of protein, relative to the needs of mice. Thus, the $12 \%$ protein diet more appropriately represents "a diet containing a sufficient amount of protein". Secondly, differences in intestinal microbiota between the $3 \%$ and $12 \%$ group at baseline were observed; we sought to randomize individual mice so average body weight became comparable under each diet. The reason of differences at the baseline could be that intestinal microbiota changed non-directionally during this period in mice of each cage. To confirm if this change in intestinal microbiota during the acclimatization period resulted in discordance in intestinal microbiota at day 0 of experiment among cages, we calculated weighted UniFrac distance at baseline among mice of 3\% protein group $(n=5)$, among mice of $12 \%$ protein group $(n=5)$ and among mice of $3 \%$ and $12 \%$ protein groups together $(n=10)$ (Supplementary Figure S6). It was revealed that discordance in intestinal microbiota observed in mice of $3 \%$ and $12 \%$ protein groups together did not differ from that observed in mice of $3 \%$ protein or $12 \%$ protein group only. In other words, differences in gut microbiota between 3\% protein group and $12 \%$ group at baseline are comparable to that observed in each group.

Intestinal microbiota and their hosts are in a symbiotic relationship. Previous studies indicated that the changes in nutritional composition of the diet can affect host health via intestinal microbiota shifts ${ }^{28,29}$. In this study, we detected characteristic changes in intestinal microbiota in the $3 \%$ and $12 \%$ protein groups. Specifically, in the $3 \%$ group we detected the increase of abundance of several bacterial species suggested to be related to "urea nitrogen salvage". Further studies to clarify the roles of intestinal microbiota related to protein intake will contribute to solving challenges associated with inadequate human nutrition, particularly in low-income countries.

\section{Methods}

Animals. Male BALB/cAJcl SPF mice (9 weeks of age) were purchased from CLEA Japan Inc. (Tokyo, Japan). The mice were kept in a room at $24 \pm 1{ }^{\circ} \mathrm{C}, 55 \pm 5 \%$ relative humidity, on a 12 -h light/dark cycle. The animals were housed in cages $(n=5$ per cage) with wood shavings and fed and watered ad libitum. All experiments were approved by the Animal Care and Use Committee of the University of Tokyo. All methods were conducted in strict accordance with the guidelines regarding animal research of the University of Tokyo. 
Experimental design. In the first experiment, all mice $(\mathrm{n}=20)$ were acclimatized on a commercial solid diet (NMF; Oriental Yeast Co, Ltd., Tokyo, Japan) for 1 week. After acclimatization, we randomized mice individuals based on body weight and then divided mice into four groups and fed them a diet containing 3, 6, 9, or $12 \mathrm{~g}$ of protein/100 g dry weight (Oriental Yeast Co., Ltd). The nutritional components of each diet are shown in Supplementary Table S3. These four groups were fed in parallel. Mice were individually recognized using a picric acid-saturated solution with ethanol. Protein concentrations of experimental diet were determined based on an experiment by Nakata et al. that demonstrated lower body weight gain with a diet containing $8 \%$ protein compared to that with a $12 \%$ protein $\operatorname{diet}^{30,31}$. Body weight was individually measured weekly, and food and water consumption were measured weekly per group. After the feeding experiment for 4 weeks, the mice were euthanized using carbon dioxide. Whole blood samples were collected during exsanguination on experimental day 28 , centrifuged for $15 \mathrm{~min}$ at $1500 \times \mathrm{g}$, and the serum samples were stored at $-80^{\circ} \mathrm{C}$ until analysis.

In the second experiment, an additional 20 mice were acclimatized, randomized and divided into four groups as occurred in the first experiment. Body weight was individually measured weekly, and food and water consumption were measured weekly per group. After the feeding experiment for 4 weeks, the mice were euthanized using carbon dioxide. Fecal samples were collected from each mouse in the $3 \%$ and $12 \%$ protein diet groups directly at defecation every seven days (day $0,7,14,21$ and 28 ), and then stored at $-80^{\circ} \mathrm{C}$ until $16 \mathrm{~S}$ rRNA gene sequencing was conducted.

Sample size calculation. For estimating minimum sample size for detecting difference in body weight on day 28 , we analyzed the results of the first experiment which used 20 mice ( $n=5$ mice per group) in total. We calculated a minimum sample size for the second experiment by assuming significance level 0.05 and statistical power 0.80 . Minimum sample sizes on day 14, 21 and 28 were estimated to be 16, 16 and 15 in total, respectively (Supplementary Table S4). Based on this result, we decided to allocate 5 mice in each cage and feed different protein diet.

Serum biomarker analysis. Serum Alb concentrations were measured using the BCG method, and serum TP concentrations were measured using the biuret method following the manufacturer's instructions (A/G B-test Wako, Wako Pure Chemical, Osaka, Japan). The serum A/G ratio was calculated as Alb/(TP - Alb). BUN was measured using a urease-indophenol method (urea nitrogen-B test; Wako Pure Chemical).

Bacterial DNA extraction. 16S rRNA gene sequencing analysis was performed using fecal samples from the $3 \%$ and $12 \%$ protein diet groups at day $0,7,14,21$ and 28 . Bacterial DNA was extracted following a previously described method $^{32}$. Frozen fecal pellets were thawed and suspended in $1 \mathrm{~mL}$ of TE10 (10 mM Tris- $\mathrm{HCl}$, $10 \mathrm{mM}$ EDTA) buffer containing RNaseA (final concentration of $100 \mu \mathrm{g} / \mathrm{mL}$, NIPPON GENE, Tokyo, Japan). Lysozyme (Sigma-Aldrich Co., Tokyo, Japan) was added to a final concentration of $15 \mathrm{mg} / \mathrm{mL}$, and the suspension was incubated for $1 \mathrm{~h}$ at $37{ }^{\circ} \mathrm{C}$ with gentle mixing. Purified achromopeptidase (Wako Pure Chemical) was added to a final concentration of 2000 units $/ \mathrm{mL}$, and the suspension was further incubated for $30 \mathrm{~min}$ at $37^{\circ} \mathrm{C}$. Sodium dodecyl sulfate (final concentration $1 \mathrm{mg} / \mathrm{mL}$ ) and proteinase $\mathrm{K}$ (final concentration $1 \mathrm{mg} / \mathrm{mL}$, Merck Japan, Tokyo, Japan) were added to the sample and this mixture was incubated for $1 \mathrm{~h}$ at $55^{\circ} \mathrm{C}$. The DNA was extracted with phenol/chloroform/isoamylalcohol (25:24:1), precipitated with isopropanol and $3 \mathrm{M}$ sodium acetate, washed with $75 \%$ ethanol, and resuspended in $200 \mu \mathrm{L}$ of TE buffer. The DNA was purified with a $20 \%$ PEG solution (PEG6000 in $2.5 \mathrm{M} \mathrm{NaCl}$ ), pelleted by centrifugation, rinsed with $75 \%$ ethanol, and dissolved in TE buffer.

16S rRNA gene amplicon sequencing. The V1-V2 region of the bacterial $16 \mathrm{~S}$ rRNA gene was amplified by polymerase chain reaction (PCR) using the universal primers 27Fmod (5'-AGRGTTTGATYMTGGCTC AG-3') and 338R (5'-TGCTGCCTCCCGTAGGAGT-3') as described previously ${ }^{33}$. Amplicons generated from each sample $(\sim 330 \mathrm{bp})$ were subsequently purified using AMPure XP (Beckman Coulter). DNA was quantified using a Quant-iT Picogreen dsDNA assay kit (Invitrogen) and a TBS-380 Mini-Fluorometer (Turner Biosystems). The $16 \mathrm{~S}$ rRNA gene sequencing was performed with the MiSeq sequencing system (Illumina, San Diego, CA, USA) according to Illumina protocol.

Data analysis. Two paired-end reads were merged using the fastq-join program. The reads lacking both forward and reverse primer sequences were removed using blast followed by trimming off both primer sequences. The reads with average quality values $<25$ were further removed. Among these high-quality reads, 3000 reads/ sample were randomly selected and were subjected to operational taxonomic unit clustering and UniFrac analysis. The selected reads of all the samples were firstly sorted by frequency of redundant sequences and grouped into operational taxonomic units (OTUs) using UCLUST (https://www.drive5.com/) with a sequence identity threshold of $97 \%$. The sequence with the highest redundancy in each OTU was determined as a representative sequence. The representative sequences of the OTUs were subjected to homology search against the following $16 \mathrm{~S}$ databases using the GLSEARCH program for taxonomic assignments. For the assignment at the phylum, genus, and species levels, sequence similarity thresholds of $70 \%, 94 \%$ and $97 \%$, respectively, were applied. The $16 \mathrm{~S}$ database was reconstructed from three publicly available databases: Ribosomal Database Project (RDP) v. 10.27, CORE (https://microbiome.osu.edu/), and a reference genome sequence database obtained from the NCBI FTP site (ftp://ftp.ncbi.nih.gov/genbank/, December 2011).

The richness of the microbiota was estimated by the Chaol and ACE indices ${ }^{34,35}$. Shannon's index was calculated to assess diversity ${ }^{36}$. PCoA was performed based on UniFrac distances. 
Statistical analysis. All statistical analyses were conducted with the R software program (v3.5.1). Body weight measured during the experiment was expressed as raw body weight. Impact of protein content in diet on body weight and serum biomarkers were investigated by using one-way ANOVA and Tukey-Kramer post hoc tests. Difference in water and food consumption by type of diet could not be tested statistically because they were measured by cage and individual variation among mice in a cage were not available.

Wilcoxon rank sum test was used for comparison of alpha diversities and the proportion of intestinal bacteria at the phylum, genus, and OTU levels. PERMANOVA was used to compare the intestinal microbiota structure among groups based on both weighted and unweighted UniFrac distance ${ }^{37}$. $P$ values were corrected for multiple testing using the Benjamini-Hochberg method as appropriate.

\section{Data availability}

All data generated or analyzed during this study are included in this published article and its supplementary information files. The output data generated by next-generation sequencing in this paper have been submitted to the DDBJ Sequence Read Archive (DRA) under the accession number DRA009131 and DRA009688.

Received: 28 November 2019; Accepted: 21 September 2020

Published online: 13 October 2020

\section{References}

1. Velisek, J. The Chemistry of Food 40-41 (Wiley, New York, 2014).

2. Schaible, U. E. \& Stefan, H. E. Malnutrition and infection: complex mechanisms and global impacts. PLoS Med. 4, e115. https:// doi.org/10.1371/journal.pmed.0040115 (2007).

3. Cicely, D. W. A nutritional disease of childhood associated with a maize diet. Arch. Dis. Child. 8, 423-433 (1933).

4. van Zutphen, T. et al. Malnutrition-associated liver steatosis and ATP depletion is caused by peroxisomal and mitochondrial dysfunction. J. Hepatol. 65, 1198-1208 (2016).

5. Ahmed, T., Rahman, S. \& Cravioto, A. Oedematous malnutrition. Indian J. Med. Res. 130, 651-654 (2009).

6. Batool, R., Butt, M. S., Sultan, M. T., Saeed, F. \& Naz, R. Protein-energy malnutrition: A risk factor for various ailments. Crit. Rev. Food Sci. Nutr. 55, 242-253 (2015).

7. Protein-Energy Ratios, Joint FAO/WHO/UNU Expert Consultation on Energy and Protein Requirements, Rome, p. 5-17 (1981).

8. Sumesh, P. S. et al. Effect of PEM on thyroid status, serum total protein and A/G ratio in pre-school going children. IJRMS. 5, 4486-4489 (2017).

9. Smith, M. I. et al. Gut microbiomes of Malawian twin pairs discordant for kwashiorkor. Science 339, 548-554 (2013).

10. Blanton, L. V. et al. Gut bacteria that prevent growth impairments transmitted by microbiota from malnourished children. Science. 351, aad3311 (2016).

11. Faith, J. J., McNulty, N. P., Rey, F. E. \& Gordon, J. I. Predicting a human gut microbiota's response to diet in gnotobiotic mice. Science 333, 101-104 (2011).

12. Larson, K. R. et al. Sex differences in the hormonal and metabolic response to dietary protein dilution. Endocrinology 158(10), 3477-3487 (2017).

13. Chaumontet, C. et al. Low-protein and methionine, high-starch diets increase energy intake and expenditure, increase FGF21, decrease IGF-1, and have little effect on adiposity in mice. Am. J. Physiol. Regul. Integr. Comp. Physiol. 316(5), R486-R501 (2019).

14. Laeger, T. et al. FGF21 is an endocrine signal of protein restriction. J. Clin. Investig 124(9), 3913-3922 (2014).

15. Kuwahata, M., Hasegawa, M., Kobayashi, Y., Wada, Y. \& Kido, Y. An oxidized/reduced state of plasma albumin reflects malnutrition due to an insufficient diet in rats. J. Clin. Biochem. Nutr. 60(1), 70-75 (2017).

16. Wada, Y. et al. Increased Ratio of Non-mercaptalbumin-1 Among Total Plasma Albumin Demonstrates Potential Protein Undernutrition in Adult Rats. Front. Nutr. 5, 64. https://doi.org/10.3389/fnut.2018.00064 (2018).

17. Hubert, M. F., Laroque, P., Gillet, J. P. \& Keenan, K. P. The effects of diet, ad Libitum feeding, and moderate and severe dietary restriction on body weight, survival, clinical pathology parameters, and cause of death in control Sprague-Dawley rats. Toxicol. Sci. 58(1), 195-207. https://doi.org/10.1093/toxsci/58.1.195 (2000).

18. Hoffmann, H. et al. Description of Enterobacter ludwigii sp. Nov., a novel Enterobacter species of clinical relevance. Syst. Appl. Microbiol. 28(3), 206-212 (2005).

19. Jalowiecki, L. et al. Bacteria from on-site wastewater treatment facilities as enzymes producers for applications in environmental technologies in Environmental Engineering V (ed. Malgorzata P and Lucjan P) 118-120 (CRC Press, Boca Raton, 2018).

20. Bardiya, N. \& Bae, J. H. Role of Citrobacter amalonaticus and Citrobacter farmeri in dissimilatory perchlorate reduction. J. Basic Microbiol. 44, 88-97 (2004).

21. Bernard, K. A. et al. Assignment of Brevibacterium stationis (ZoBell and Upham 1944) Breed 1953 to the genus Corynebacterium, as Corynebacterium stationis comb. nov., and emended description of the genus Corynebacterium to include isolates that can alkalinize citrate. Int. J. Syst. Evol. Microbiol. 60, 874-879 (2010).

22. Stepanović, S. et al. Supplementary biochemical tests useful for the differentiation of oxidase positive staphylococci. Syst. Appl. Microbiol. 30(4), 316-318 (2007).

23. Spergser, J. et al. Staphylococcus nepalensis sp. nov, isolated from goats of the Himalayan region. Int. J. Syst. Evol. Microbiol. 53(6), 2007-2011 (2003).

24. Stewart, G. S. \& Smith, C. P. Urea nitrogen salvage mechanisms and their relevance to ruminants, non-ruminants and man. Nutr. Res. Rev. 18, 49-62 (2005).

25. Takahashi, M., Benno, Y. \& Mitsuoka, T. Utilization of ammonia nitrogen by intestinal bacteria isolated from pigs. Appl. Environ. Microbiol. 39, 30-35 (1980).

26. Castell, D. O. \& Edward, W. M. Ammonia absorption from the human colon: The role of nonionic diffusion. Gastroenterology 60(1), 33-42 (1971).

27. Bisanz, J. E. et al. Meta-analysis reveals reproducible gut microbiome alterations in response to a high-fat diet. Cell Host Microbe. 26(2), 265-272 (2019).

28. Rasnik, K. S. et al. Infuence of diet on the gut microbiome and implications for human health. J. Transl. Med. 15, 73. https://doi. org/10.1186/s12967-017-1175-y (2017).

29. Lee, Y. K. Effects of diet on gut microbiota profile and the implications for health and disease. Biosci. Microbiota Food Health 32(1), $1-12(2013)$.

30. Nakata, S. \& Kimura, K. Availability of mice in protein nutrition study. J. Soc. Nutr. Food Sci. 40, 111-116 (1987).

31. Bing, F. C., Adams, W. L. \& Bowman, R. O. The protein requirements of the albino mouse. J. Nutr. 5(6), 571-579 (1932).

32. Morita, H. et al. An improved DNA isolation method for metagenomic analysis of the microbial flora of the human intestine. Microbes Environ. 22, 214-222 (2007). 
33. Kim, S. W. et al. Robustness of gut microbiota of healthy adults in response to probiotic intervention revealed by high-throughput pyrosequencing. DNA Res. 20, 241-253 (2013).

34. Chao, A. Nonparametric estimation of the number of classes in a population. Scand. J. Stat. 11, 265-270 (1984).

35. Chao, A. \& Lee, S. M. Estimating the number of classes via sample coverage. J. Am. Stat. Assoc. 87, 210-217 (1992).

36. Shannon, C. E. A mathematical theory of communication. Bell Syst Tech. J. 27, 379-423 (1948).

37. Lozupone, C., Lladser, M. E., Knights, D., Stombaugh, J. \& Knight, R. UniFrac: An effective distance metric for microbial community comparison. ISME J. 5, 169-172 (2011).

\section{Acknowledgements}

Our work was supported in part by a Grant-in-Aid for Scientific Research (15H04430, 19H03315) and Takeda Science Foundation.

\section{Author contributions}

H.M., W.S., P.H., A.G. M.U. and K.H. conceived the experiments, H.M., E.T. and K.H. conducted experiments, H.M., W.S., E.T., C.S., L.T. and M.H. analyzed data. All authors reviewed the manuscript.

\section{Competing interests}

The authors declare no competing interests.

\section{Additional information}

Supplementary information is available for this paper at https://doi.org/10.1038/s41598-020-74122-9.

Correspondence and requests for materials should be addressed to H.M. or W.S.

Reprints and permissions information is available at www.nature.com/reprints.

Publisher's note Springer Nature remains neutral with regard to jurisdictional claims in published maps and institutional affiliations.

(c) (i) Open Access This article is licensed under a Creative Commons Attribution 4.0 International License, which permits use, sharing, adaptation, distribution and reproduction in any medium or format, as long as you give appropriate credit to the original author(s) and the source, provide a link to the Creative Commons licence, and indicate if changes were made. The images or other third party material in this article are included in the article's Creative Commons licence, unless indicated otherwise in a credit line to the material. If material is not included in the article's Creative Commons licence and your intended use is not permitted by statutory regulation or exceeds the permitted use, you will need to obtain permission directly from the copyright holder. To view a copy of this licence, visit http://creativecommons.org/licenses/by/4.0/.

(C) The Author(s) 2020 